\title{
Tratamiento con aparatos ortopédicos fijos y su infiuencia en el estado nutricional e ingesta dietética en preescolares con labio y paladar hendido
}

Pérez-Muñoz Paulina Adriana, ${ }^{*}$ Pedroza-García Karina Alejandra, ${ }^{* *}$ Masuoka-Ito David, ${ }^{* * *}$ Martín del Campo-Cervantes Judith, ${ }^{* *}$ Ramírez-Orozco Ricardo Ernesto**

\section{Resumen}

- Antecedentes: el labio leporino y el paladar hendido (CLP) es - una de las alteraciones congénitas más comunes que afecta las - estructuras faciales y, en consecuencia, interfiere con el proceso - de alimentación y un estado nutricional adecuado. El tratamien- to ortopédico puede desempeñar un papel en los trastornos nu- tricionales y dietéticos. Objetivo: evaluamos el estado nutricional

- y la ingesta dietética en niños en edad preescolar con CLP bajo

- el tratamiento de dispositivos ortopédicos fijos. Métodos: estudio

- transversal en 31 niños (3 a 6 años) con CLP; se tomaron me-

- didas antropométricas bajo los criterios de los puntajes Z de la

- Organización Mundial de la Salud, así como el recuerdo dietético

- de 24 horas para la evaluación dietética (macro y micronutrien-

- tes). Resultados: $19.4 \%$ de los niños tenían bajo peso y $25.8 \%$

- presentaron sobrepeso u obesidad; se registró una dieta insufi-

- ciente en nutrientes específicos, tales como: fibra, ácido fólico,

- hierro, potasio y fósforo. La adherencia al tratamiento con dispo-

- sitivos ortopédicos se asoció con una baja ingesta de proteínas,

- además de un mayor consumo de lípidos en aquellos que siguen

- una correcta adherencia al tratamiento. Conclusiones: los niños

- con CLP pueden comprometer sus requerimientos de dieta y, en

- consecuencia, su estado nutricional. El material y funcionalidad

- de los dispositivos ortopédicos deben considerarse para lograr

- una rehabilitación adecuada y un crecimiento normal. LUXMÉDI-

- CA AÑO 16, NÚMERO 46, ENERO-ABRIL 2021, PP. 19-28.

Palabras clave: aparatos ortopédicos fijos, estado nutricio, dieta, ingesta nutricional, labio y paladar hendido.

\section{Abstract}

Background: Cleft lip and palate (CLP) is one of the most common congenital disorders that affect facial structures and, consequently, interferes with the feeding process and an adequate nutritional status. Orthopedic treatment can play a role in nutritional and dietary disorders. Objective: We evaluated the nutritional status and dietary intake in preschool children with CLP treated with fixed orthopedic devices. Methods: This is a cross-sectional study, including 31 children (3 to 6 years) with CLP. Anthropometric measurements were taken following the criteria of the Z-score of the World Health Organization and the 24-hour dietary recall for the dietary evaluation (macro and micronutrients). Results: 19.4\% of the children were underweight, and $25.8 \%$ were overweight or obese. A diet insufficient in specific nutrients was recorded, such as fiber, folic acid, iron, potassium, and phosphorus. Adherence to treatment with orthopedic devices was associated with a low protein intake and a higher lipid intake in those who follow correct adherence to treatment. Conclusions: Children with CLP may compromise their dietary requirements and, consequently, their nutritional status, material and functionality of the orthopedic devices must be considered to achieve adequate rehabilitation and normal growth. LUXMÉDICA AÑO 16, NÚMERO 46, ENERO-ABRIL 2021, PP. 19-28.
Keywords: fixed orthopedic devices, nutritional status, diet, nutritional intake, cleft lip and palate

Pérez-Muñoz Paulina Adriana. Departamento de Nutrición, Centro Estatal de Atención para Labio y Paladar Hendido, Aguascalientes, México. https://orcid.org/0000-0001-8461-0882. Correo electrónico: paulinni_10@hotmail.com

** Pedroza-García Karina Alejandra. Departamento de Nutrición, Centro de Ciencias de la Salud Universidad Autónoma de Aguascalientes, \#20131. Aguascalientes, México. https://orcid.org/0000-0001-6428-1743. Correo electrónico: Inkarina.pedroza@hotmail.com

** Martín del Campo-Cervantes Judith. Profesora investigadora de tiempo completo del Departamento de Nutrición del Centro de Ciencias de la Salud de la Universidad Autónoma de Aguascalientes. https://orcid.org/0000-0003-1855-6309. Correo electrónico: judith.martindelcampo@edu.uaa.mx

** Ramírez-Orozco Ricardo Ernesto. Profesor investigador de tiempo completo del Departamento de Nutrición del Centro de Ciencias de la Salud de la Universidad Autónoma de Aguascalientes. https://orcidorg/0000-0001-6175-0698.Correo electrónico: dcmrero@gmail.com.

*** Masuoka-lto David. Profesor investigador de tiempo completo del Departamento de Estomatología del Centro de Ciencias de la Salud de la Universidad Autónoma de Aguascalientes. https:/orcid.org/0000-0002-4850-4342.Correo electrónico: dimasuoka@correo.uaa.mx

Fecha de recibido: 2 de marzo 2020

Fecha de aceptación: 30 de julio 2020

Correspondencia: Dr. Ricardo Ernesto Ramírez Orozco. Secretaría de Investigación y Posgrado. Edificio 101, planta alta. Ciudad Universitaria. Avenida Universidad 940. Código postal 20131. Aguascalientes, Ags, México. Teléfono 4499107400 extensión 53014. Correo electrónico: dcmrero@gmail.com. 


\section{Introducción}

Los defectos del labio leporino y el paladar hendido (CLP) constituyen una de las alteraciones congénitas más comunes que afectan las estructuras faciales. Esta afección puede involucrar huesos y tejidos blandos, comenzando desde los labios hasta las fisuras del paladar que se conectan con la cavidad nasal provocando daños en los oídos y los ojos. ${ }^{1}$ El CLP afecta aproximadamente a 1 de cada 700 nacimientos, la incidencia varía según la zona geográfica, las características raciales y étnicas, la exposición ambiental y el estado socioeconómico; esta condición se clasifica como sindrómica (SCLP) y paladar hendido no sindrómico (NSCLP) según la presencia de anomalías. La tasa de prevalencia NSCLP es superior al 70\% de los casos de hendidura oro-facial (figura 1).
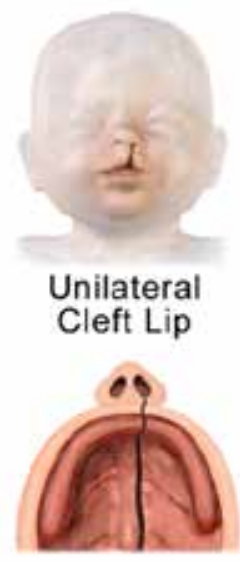

Unilateral Cleft Lip and Palate
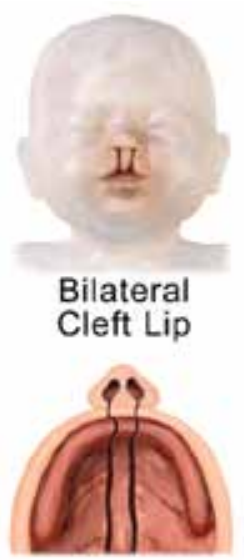

Bilateral Cleft Lip and Palate

Fig. 1 Clasificación de labio y paladar hendido.

Fuente: Creative Commons Attribution-Share Alike 4.0 International; Autor: Bruce Blaus.

El CLP implica problemas de alimentación, alteraciones de la comunicación y el comportamiento, baja autoestima y desarrollo de una relación deficiente con la sociedad. ${ }^{3}$ Los avances ortopédicos en el tratamiento han brindado opciones para pacientes con CLP, tales como: placa Schwarz, mascarilla facial, placa Haas, son ejemplos de dispositivos funcionales que pueden ayudar a reducir las alteraciones estéticas y funcionales. ${ }^{4}$ Los niños con hendidura facial congénita tienen problemas de alimentación y nutrición relacionados con sus diferencias anatómicas. ${ }^{5}$ La regurgitación nasal y la protección inadecuada de las vías respiratorias durante la deglución pueden provocar neumonía y daño pulmonar. Por otro lado, el tratamiento nutricional como la lactancia materna es vital para mejorar y recuperar un crecimiento adecuado y, en consecuencia, facilitar su proceso de alimentación. ${ }^{6}$ 
Un estudio de Wysocka y cols. acerca de los dispositivos ortopédicos y su influencia en la ingestión de alimentos, reporta que un alto porcentaje de niños con dispositivos fijos no cumplen con sus requerimientos nutricionales diarios. ${ }^{7}$ Se requiere educación dietética y nutricional para mejorar el estado nutricional y prevenir las consecuencias de la desnutrición en pacientes con CLP. El objetivo de este estudio fue evaluar la dieta y el estado nutricional en niños en edad preescolar con labio leporino y paladar hendido bajo el tratamiento de dispositivos ortopédicos fijos.

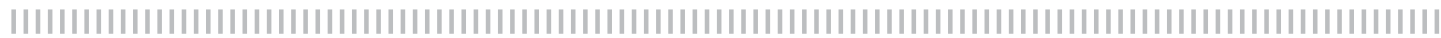

\section{Material y métodos}

Muestra: el diseño fue un estudio descriptivo de corte transversal; la selección de la muestra y las evaluaciones se llevaron a cabo de enero a julio de 2018, estudiamos una muestra de 31 niños (3-6 años) con CLP en tratamiento ortopédico con dispositivos fijos. Los participantes fueron pacientes del Centro Estatal de Atención para Labio y Paladar Hendido, en Aguascalientes, México. Se obtuvo el consentimiento informado de los padres de los participantes.

Evaluación antropométrica y dietética: el peso y la altura se midieron con una escala modelo SECA ${ }^{\circledR} 700$ y un estadiómetro estable modelo SECA ${ }^{\circledR} 217$, todas las medidas fueron tomadas por duplicado por un nutricionista estandarizado de acuerdo con la técnica de la OMS. Se utilizaron para la evaluación los estándares de crecimiento infantil de las puntuaciones $z$ de la OMS para: peso para la edad $(\mathrm{P} / \mathrm{E})$, talla para la edad (T/E) y el índice de masa corporal para la edad (IMC). Se utilizó el software de la versión $W H O$ AnthroPlus ${ }^{\circledR}$ 1.0.4 para los cálculos. ${ }^{8}$ Los puntajes Z comienzan por debajo de -2 , resultados como bajo peso, altura baja o desnutrición; comenzando por encima de +2 se consideraron sobrepeso $\mathrm{u}$ obesidad. Para la evaluación dietética, se obtuvieron tres recordatorios dietéticos de 24 horas de padres que consideraron dos días seguidos entre semana y uno el fin de semana. Los datos se analizaron con el software Az Nutrition $\AA$. Se calcularon macro y micronutrientes y se compararon con la ingesta dietética recomendada (IDR) para la población mexicana; menos del $90 \%$ de la IDR se consideró una ingesta deficiente y más del $110 \%$ un consumo excesivo.

Análisis estadístico: los datos se muestran como media, desviación estándar, mediana e intervalo intercuartílico (IQR) o porcentaje, según corresponda. La normalidad se evaluó con la prueba de ShapiroWilks. Comparamos grupos (adherencia al tratamiento / no adherencia al tratamiento) utilizando la prueba U Mann-Whitney para variables independientes. Se utilizó la correlación de rango de Spearman y la prueba post hoc para la posible asociación entre la dieta y el cumplimiento del tratamiento. Todos los datos se analizaron con el software de versión SPSS 23.0 para Windows. La significancia estadística se consideró a partir de $p<0,05$.

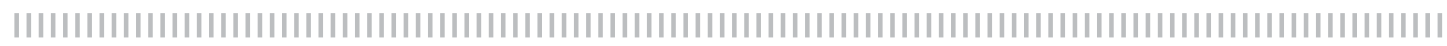

\section{Resultados}

\section{Características generales de la población estudiada}

Se evaluaron 31 niños (16 niñas / 15 niños) con una edad promedio de $4.8 \pm 1.2$ años, las características generales se muestran en la tabla 1. El 71\% de los niños tenía labio leporino y paladar hendido unilateral (UCLP), 19.4\% CLP bilateral (BCLP), 3.2\% paladar hendido $(\mathrm{CP})$ y $6.5 \%$ labio leporino $(\mathrm{CL})$. En relación con el tratamiento ortopédico: el 
$58.1 \%$ usó placa Schwarz; el 16.1\%, máscara facial; y el 12.9\%, placa Haas; el 10\% de los niños usaron más de un dispositivo.

\section{Tabla I}

\section{Características generales de niños con CLP}

\begin{tabular}{|l|c|c|c|}
\hline Variable & Media \pm ES & Mínimo & Máximo \\
\hline Edad (años) & $4.80 \pm 1.2$ & 3.3 & 6.9 \\
\hline Peso (kg) & $17.80 \pm 3.4$ & 13.4 & 24 \\
\hline Talla (cm) & $107.03 \pm 9.2$ & 92 & 128 \\
\hline IMC (kg/m2) & $15.42 \pm 1.8$ & 12.9 & 20.1 \\
\hline
\end{tabular}

Datos son representados como media y desviación estándar (DE).IMC: indice de masa corporal. $(n=31)$

\section{Estado nutricional y labio leporino y paladar hendido}

Se encontró desnutrición en el $29.1 \%$ de los niños; el $19.4 \%$ de ellos tenían bajo peso, mientras que el $9.7 \%$ tenían sobrepeso u obesidad según los puntajes z P/E. Por otro lado, el 22,6\% presentaba una altura corta (retraso en el crecimiento) según los criterios T/E. Finalmente, al evaluar el IMC, aproximadamente el $20 \%$ tenía desnutrición o bajo peso; sin embargo, el $25.8 \%$ se clasificó con sobrepeso u obesidad (figura 2), discrepancias entre P/E e IMC. Finalmente, se observó una mejor condición nutricional en los niños que no se adhirieron al tratamiento ortopédico, lo que significa un mayor aumento de peso (tabla 2).

\section{Tabla 2}

\section{Evaluación del estado nutricio y su adherencia al tratamiento ortopédico}

\begin{tabular}{|l|c|c|c|}
\hline Variable & $\begin{array}{c}\text { Adherencia al trata- } \\
\text { miento ortopédico } \\
(\mathbf{n = 2 1})\end{array}$ & $\begin{array}{c}\text { Sin adherencia al tra- } \\
\text { tamiento ortopédico } \\
(\mathbf{n = 1 0 )}\end{array}$ & $\mathbf{P}$ \\
\hline P/E (Z-score) & $0.13(-0.55,0.77)$ & $-0.86(-1.29,-0.27)$ & 0.004 \\
\hline T/E (Z-score) & $0.03(-0.95,0.75)$ & $-0.88(-1.04,0.20)$ & 0.287 \\
\hline IMC-para-Edad (Z-score) & $0.57(-0.87,1.32)$ & $-0.95(-1.63,-0.24)$ & 0.031 \\
\hline
\end{tabular}

Datos representados como mediana y rango intercuartil (IQR: 25-75). P / E Peso para la edad, T / E: Talla para la edad, IMC: índice de masa corporal. Se utilizó la prueba U Mann-Whitney, nivel de significancia: $p$ $<0,05$. 

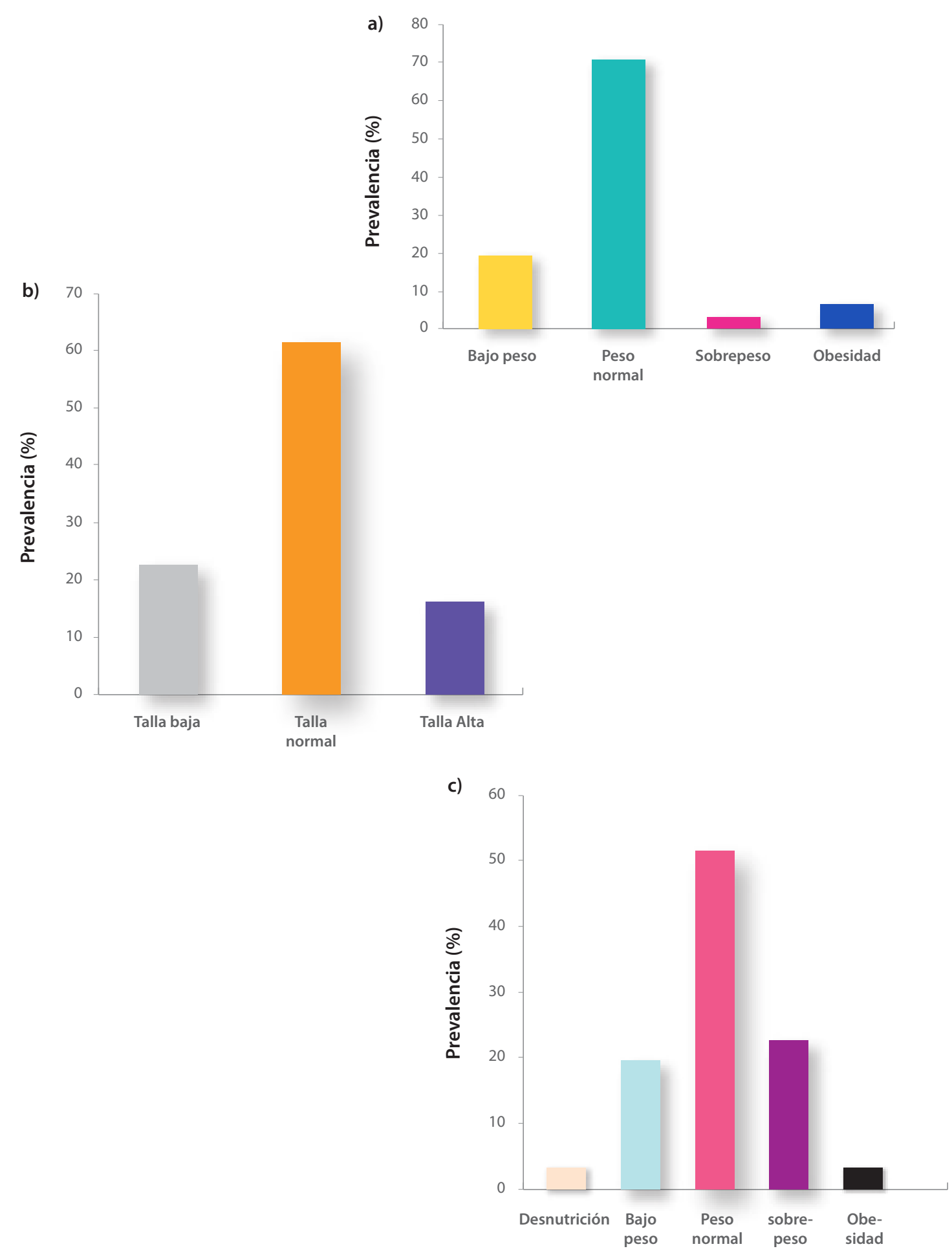

Fig. 2: Evaluación antropométrica de preescolares con CLP.

a) Estado nutricional basado en el indicador de peso para la edad, b) estado nutricional basado en el indicador de talla para la edad y c) estado nutricional basado en el indicador de índice de masa corporal para la edad. $(n=31)$ 


\section{Ingesta dietética versus ingesta dietética recomendada (IDR) en CLP}

Los resultados de la evaluación dietética fueron los siguientes: la ingesta calórica fue de $1.431,12 \pm 268,7 \mathrm{kcal} /$ día. Se calcularon macronutrientes y micronutrientes (tabla 3 y 4): carbohidratos (188.96 $\pm 38.9 \mathrm{~g} /$ día), proteínas $(52.42 \pm 11.3 \mathrm{~g} /$ día $)$ y lípidos (51.74 $\pm 15.25 \mathrm{~g} / \mathrm{día}$ ). Varias deficiencias en la ingesta de micronutrientes estuvieron presentes en los niños con CLP. Cabe señalar que la edad marca una diferencia en la falta o exceso de ingesta de nutrientes en la dieta, pero en general los resultados mostraron un consumo deficiente de fibra, ácido fólico, hierro, potasio y fósforo según sus requisitos de edad.

\section{Tabla 3}

\section{Evaluación dietética en niños con CLP}

\begin{tabular}{|c|c|c|c|}
\hline Variable & Media $\pm \mathrm{DE}$ & Mínimo & Máximo \\
\hline Energía (kcal) & $1431.1 \pm 268.7$ & 1006.9 & 2242.2 \\
\hline Carbohidratos(g) & $188.9 \pm 38.9$ & 135.1 & 312.1 \\
\hline Proteínas (g) & $52.4 \pm 11.3$ & 33.1 & 84.3 \\
\hline Lípidos (g) & $51.7 \pm 15.2$ & 27.4 & 99.7 \\
\hline Colesterol (mg) & $210.5 \pm 91.2$ & 83.2 & 518.9 \\
\hline Fibra (g) & $11.1 \pm 4.4$ & 4.5 & 24.1 \\
\hline Azúcar (g) & $55.8 \pm 32.5$ & 16 & 161.5 \\
\hline Índice glucémico (g) & $404.1 \pm 187$ & 69.3 & 1122.3 \\
\hline Carga glucémica (g) & $129.7 \pm 105.6$ & 14.6 & 594.6 \\
\hline Vitamina A $(\mu g)$ & $458.1 \pm 736.4$ & 79.7 & 4258.8 \\
\hline Ácido fólico( $\mu \mathrm{g})$ & $95.4 \pm 61.6$ & 23.7 & 316.2 \\
\hline Vitamina C (mg) & $39.3 \pm 37.6$ & 0.1 & 145.6 \\
\hline Hierro No-Hem (mg) & $0.8 \pm 0.6$ & 0.1 & 2.8 \\
\hline Hierro Hem (mg) & $7.4 \pm 4.6$ & 1.7 & 27.8 \\
\hline Potasio (mg) & $389.6 \pm 280.2$ & 1.2 & 1066 \\
\hline Sodio (mg/d) & $1161.1 \pm 455.2$ & 628.2 & 2701 \\
\hline Calcio (mg) & $826.6 \pm 301.6$ & 343.8 & 1741.5 \\
\hline Selenio (mg) & $19.4 \pm 10.6$ & 4.5 & 44.4 \\
\hline Fósforo (mg) & $33.2 \pm 68.3$ & 0.1 & 336.3 \\
\hline
\end{tabular}

DE: Desviación estándar. $(n=31)$ 


\section{Tabla 4}

Ingesta de micronutrientes acorde a la edad

\begin{tabular}{|c|c|c|c|c|c|c|}
\hline Variable & $<4$ años & IDR & $p$ & 4 a 6 años & IDR & $p$ \\
\hline Fibra (g) & $10 \pm 3^{a}$ & 14 & 0.004 & $11.5 \pm 4.8^{a}$ & 18 & 0.0001 \\
\hline Vitamina $\mathrm{A}(\mu \mathrm{g})$ & $352.3 \pm 277.5$ & 300 & 0.5 & $501.3 \pm 859.4$ & 400 & 0.5 \\
\hline Ácido fólico $(\mu \mathrm{g})$ & $86.3 \pm 32.6^{a}$ & 168 & 0.0001 & $99.2 \pm 70.5^{a}$ & 230 & 0.0001 \\
\hline Vitamina $C(\mathrm{mg})$ & $49.7 \pm 37.6^{b}$ & 15 & 0.02 & $35 \pm 37.7$ & 25 & 0.2 \\
\hline Hierro (mg) & $5.2 \pm 1.6^{a}$ & 13 & 0.0001 & $8.3 \pm 5.1^{a}$ & 15 & 0.0001 \\
\hline Potasio (mg) & $554.7 \pm 299.1^{a}$ & 800 & 0.03 & $322 \pm 248.2^{a}$ & 3800 & 0.0001 \\
\hline Sodio (mg) & $915.3 \pm 158.6^{a}$ & 1100 & 0.008 & $1261.6 \pm 500$ & 1200 & 0.5 \\
\hline Calcio (mg) & $741 \pm 257.9^{b}$ & 500 & 0.02 & $861.5 \pm 316.5$ & 800 & 0.32 \\
\hline Selenio (mg) & $17.5 \pm 9.7$ & 20 & 0.47 & $20.2 \pm 11.1^{a}$ & 30 & 0.001 \\
\hline Fósforo (mg) & $19.2 \pm 30.1^{a}$ & 460 & 0.0001 & $78.7 \pm 16.8^{a}$ & 500 & 0.000 \\
\hline
\end{tabular}

Datos representados como: media y desviación estándar; IDR: ingesta dietética recomendada; ${ }^{a}$ ingesta deficiente según el porcentaje de adecuación; ${ }^{b}$ ingesta excesiva según porcentaje de adecuación. Prueba estadística utilizada: una muestra de prueba T de Student, significancia: $p<0,05$.

\section{Consumo de grasas en la dieta en CLP}

Otro hallazgo en nutrientes fue la cantidad de lípidos en la dieta, se registró una mayor ingesta en los niños (55,8 g / día frente a 45,4 g / día) en comparación con las niñas, lo mismo que el colesterol (251,2 mg / día frente a 149,4 mg / día) (Tabla 5).

\section{Tabla 5}

\section{Ingesta de grasa en niños con CLP de acuerdo con su sexo}

\begin{tabular}{|l|c|c|c|}
\hline \multicolumn{1}{|c|}{ Variable } & Niñas $(\mathbf{n}=16)$ & Niños $(\mathbf{n}=15)$ & p \\
\hline Lípidos $(\mathrm{kcal})$ & $408.9(330.2,451.4)$ & $502.17(418.9,560.3)$ & $\mathbf{0 . 0 1}$ \\
\hline Lípidos $(\mathrm{g})$ & $45.4(36.6,50.1)$ & $55.8(46.5,62.2)$ & $\mathbf{0 . 0 1}$ \\
\hline Colesterol $(\mathrm{mg})$ & $149.4(126,232.4)$ & $251.2(183.2,267)$ & $\mathbf{0 . 0 2}$ \\
\hline
\end{tabular}

Datos representados como mediana y rango intercuartil (IQR: 25-75). Se utilizó la prueba U-Mann Whitney, nivel de significancia: $p<0.05$. $(n=31)$

\section{Adherencia al tratamiento ortopédico: ¿una consideración para un alimento específico?}

Se encontró una correlación inversa entre el contenido calórico en la dieta $(R=-0.442, p$ $=0.01)$ y la ingesta de proteínas $(R=-0.435, p=0.01)$ con la adherencia a dispositivos ortopédicos fijos. Esto significa que mientras hubo más adherencia al tratamiento, menor fue el consumo de proteína. 


\section{Discusión}

Se sabe que la desnutrición es una de las causas más importantes de altas tasas de morbilidad en niños en edad preescolar, que afecta a aproximadamente del $20 \%$ al 30\% de los niños de países en desarro1lo. ${ }^{9}$ Los niños con CLP sufren dificultades de alimentación y, por lo tanto, problemas de aumento de peso o incluso mantienen un peso saludable debido a sus procesos alterados de alimentación y deglución; hoy en día, se ha sugerido el uso de diversas placas con diferentes tipos de materiales que podrían facilitar su proceso de alimentación. ${ }^{10}$

Miranda y cols. demostraron que la mayoría de los niños con CLP de Brasil tenían problemas de crecimiento P/E e IMC; sin embargo, los participantes presentaron una recuperación espontánea en la primera infancia. ${ }^{11}$ Nuestros resultados son contrastantes considerando que sólo el $22 \%$ tenía poca estatura y sólo el $3.2 \%$ presentaba desnutrición, además de que muchos participantes tenían sobrepeso u obesidad. Una explicación de esto podría deberse a que México se encuentra actualmente en la cima de los países con más obesidad infantil. ${ }^{12}$ Otros autores como Gopinath y cols. estudiaron la ingesta dietética de niños (de 2 a 6 años) con CLP en Malasia, su ingesta calórica y de macronutrientes fue similar a nuestros resultados (1,337.5 kcal. vs 1,431.1 kcal.), sin mostrar diferencias con la IDR de niños sin CLP en aquellos rangos de edad. ${ }^{13}$ Nuestros resultados marcan una ingesta deficiente en algunos micronutrientes, y es sabido que esto puede afectar el desarrollo del crecimiento, el soporte de la masa muscular y el tejido óseo, explicado por la deficiencia de nutrientes específicos como el calcio y el fósforo, como ha sido publicado por algunos autores; ${ }^{14,15}$ sin embargo, la ingesta de calcio estaba bien cubierta, esto podría explicarse con el consumo elevado de productos lácteos registrado por el recuerdo dietético de 24 horas, su consistencia pue- de facilitar su consumo.

La ingestión de otras vitaminas, minerales y fibras fue inferior en comparación con la IDR, probablemente debido a una disminución en la selección de la variedad de alimentos; sin embargo, esto puede ser una consecuencia de las limitaciones del proceso de alimentación causadas por dispositivos fijos del tratamiento ortopédico ya que la textura de algunos alimentos puede dificultar la masticación y la deglución, por lo que pueden evitar consumir este tipo de alimentos.

Finalmente, la falta de adherencia al uso del dispositivo ortopédico puede ser un factor de riesgo de bajo peso, esto podría explicarse por la selección de alimentos en la dieta, los alimentos fibrosos como la carne y algunas verduras causarán un problema, el CLP afecta una masticación adecua$\mathrm{da}$, esto cambiará patrones alimentarios en niños con CLP y, en consecuencia, eligen alimentos de textura fácil, tales como: alimentos con alto contenido en grasas y alto contenido de azúcar, dando una posible explicación de nuestros resultados, la mayoría de los niños con CLP presentarán en algún momento una ingesta deficiente en la dieta, antes o durante el uso del tratamiento ortopédico con dispositivos fijos. ${ }^{7}$ Por otro lado, en el estudio de Crerand y cols. encontraron que aproximadamente el $79 \%$ de los pacientes presentaban dificultades con la adherencia al tratamiento, debido al daño de los dispositivos ortopédicos, algunas razones fueron: ruptura de dispositivos, cables, bandas o brackets. ${ }^{16}$

Entre los pacientes con problemas bucales que tienen mayor tasa de deserción en la adherencia a tratamientos ortopédicos destacan los pacientes con CLP, representando el $30 \%$ de la población que no termina el tratamiento. Actualmente no se cuenta con información sobre las razones específicas a las cuales se debe dicho abandono o poca adherencia al tratamiento; sin embargo, se mencionan y reiteran factores como higiene bucal, dolor o molestias y dificultad, 
pérdida de piezas dentales y tiempo de recuperación de heridas, tales argumentos generan incertidumbre sobre la dificultad que implica las texturas de los alimentos y la comodidad que permita desarrollar una alimentación "subnormal", viéndose reflejado en la poca adherencia de acuerdo al tipo de nutrimento ingerido. ${ }^{16-18}$

La condición de CLP por sí misma implica varios problemas. Cada paciente puede tener diferencias estructurales en la nariz y la boca impactando en la respiración mientras se alimentan. Según lo informado por Rosenberg y cols., escupir con frecuencia, regurgitar las bebidas por la nariz y el tiempo prolongado en las comidas son comunes en los niños con CLP, estos problemas normalmente son respaldados por ajustes de especialistas en hendiduras. ${ }^{19} \mathrm{La}$ influencia de la adherencia al tratamiento ortopédico en el estado nutricional de nuestros pacientes podría explicarse por la aparición de cualquiera de estos inconvenientes, mejorando la alimentación en niños que no completaron la adherencia al tratamiento.

Los niños con CLP tienen un mayor riesgo de trastornos alimenticios. Debido a este riesgo, se han sugerido intervenciones de alimentación, equipo de alimentación especializado y educación nutricional para los padres para prevenir el fracaso del crecimiento. ${ }^{20-23}$ La no adherencia al tratamiento en nuestros resultados podría aumentar la ingesta de alimentos, pero no necesariamente una ingesta adecuada de nutrientes.

\section{Conclusiones}

La ingesta dietética recomendada de la población general fue diferente en diversos nutrientes en la población con CLP. Los pacientes con adherencia al tratamiento ortopédico presentaron deficiencias en la dieta, reflejándose en el peso y la talla, esto comparado con aquellos niños que tuvieron una menor adherencia al tratamiento. Por otro lado, es de resaltar el incremento en el consumo de lípidos, esto probablemente se debe al papel que juega la textura en los alimentos con alto contenido en grasas, que tienden a ser más suaves y fáciles de consumir. Finalmente, la falta de consumo de proteína puede estar relacionado en el mismo contexto, a la textura que conllevan los alimentos cárnicos o con alto contenido de proteína, dificultando su ingesta. Es necesario evaluar la calidad de los dispositivos ortopédicos para pacientes con labio leporino y paladar hendido, una evaluación periódica nutricional y dietética debe considerarse dentro de las pautas en el tratamiento de niños con CLP que inicien tratamiento ortopédico, además de esto, es importante considerar la impartición de orientaciones nutricionales para los pacientes y padres. Esto podría apoyar la adherencia al tratamiento y a su vez mejorar una ingesta nutricional adecuada, llevando a buen término el tratamiento, reduciendo así la tasa de abandono.

\section{Contribución de los autores}

APPM realizó la evaluación dietética y nutricional de la población. Diseño de KAPG y planificación metodológica, análisis dietético y desarrollo de manuscritos. DM análisis estadístico y desarrollo del manuscrito. RERO principal revisor del estudio, análisis estadístico y desarrollo de manuscritos. 


\section{Agradecimientos}

Queremos agradecer a los padres que generosamente donaron su tiempo y esfuerzo para participar en el estudio. También agradecemos a las autoridades del Centro Estatal de Atención para Labio y Paladar Hendido que colaboraron en el estudio y por su interés en esta investigación.

\section{Fondos}

El proyecto no recibió ningún financiamiento.

\section{Conflicto de intereses}

Los autores declaran que no hay conflicto de intereses.

\section{Bibliografía}

1. Pons-Bonalsa $A$, Pons-Bonals $L$, Hidalgo-Martínez $S M$, Sosa-Ferreyra CF. Clinical-epidemiological study in children with cleft lip palate in a secondary-level hospital. Bol Med Hosp Infant Mex. 2017;74(2):107-21.

2. Crockett DJ, Goudy SL. Cleft lip and palate. Facial Plast Surg Clin North Am. 2014;22(4):573-86.

3. Roach C. Biopsychosocial Issues in Cleft Lip and Palate. Plast Surg Nurs. 2016; 36(1):28-30.

4. Tai K, Hotokezaka H, Park JH, Tai H, Miyajima K, Choi M, et al. Preliminary cone-beam computed tomography study evaluating dental and skeletal changes after treatment with a mandibular Schwarz appliance. Am J Orthod Dentofacial Orthop. 2010; 138:262.e1-262.e11.

5. Kaye A, Thaete K, Snell A, Chesser C, Goldak C, Huff H. Initial Nutritional Assessment of Infants With Cleft Lip and/or Palate: Interventions and Return to Birth Weight. Cleft Palate Craniofac J. 2017; 54(2):127-136.

6. Burca ND, Gephart SM, Miller C, Cote C. PromotingBreastMilkNutrition in InfantsWithCleftLip and/orPalate. Adv Neonatal Care. 2016;16(5):337-44.

7. Wysocka M, Cudzi?o D, Kawala B, Kopczy?ski P. Eatinghabits of patientswithcleftlip and palate treated with fixedappliances. DevPeriodMed. 2014;18(1):93-101.

8. World Health Organization: Departamento de Nutrición para la Salud y Desarrollo (2018). Curso de Capacitación sobre la Evaluación del Crecimiento del Niño. [online] Available at: http://www.who.int/childgrowth/ training/b_midiendo.pdf [Accessed 21 Aug. 2018].

9. Zamora Linares CE, Bauzá JG. Nutritional status of children suffering cleft lip and palate. Rev Cubana Pediatr. 2013; 85(3):295-300

10. Gupta R, Singhal P, Mahajan K, Singhal A. Fabricating feeding plate in CLP infants with two different materials: a series of case report. J Indian Soc Pedod Prev Dent. 2012; 30(4):352-5.

11. Miranda GS, Marques IL, de Barros SP, Arena EP, de Souza L. Weight, Length, and Body Mass Index Growth of Children Under 2 Years of Age With Cleft Lip and Palate. Cleft Palate Craniofac J. 2016; 53(3):264-71.

12. Shamah-Levy T, Cuevas-Nasu L, Gaona-Pineda EB, Gómez-Acosta LM, Morales-Rúan MDC, Hernández-Ávila $M$, et al. Overweight and obesity in children and adolescents, 2016 Halfway National Health and Nutrition
Survey update. Salud Pública Mex. 2018; 60(3):244-253.

13. Gopinath VK. Assessment of nutrientintake in cleftlip and palatechildrenaftersurgicalcorrection. Malays J MedSci. 2013;20(5):61-6.

14. Uauy R, Kain J, Mericq V, Rojas J, Corvalán C. Nutrition, child growth, and chronic disease prevention. Ann Med. 2008; 40(1):11-20.

15. Bueno AL, Czepielewski MA. The importance for growth of dietary intake of calcium and vitamin D. J Pediatr (Rio J). 2008; 84(5):386-394.

16. Crerand, C. E., Da Silveira, A. C., Kapa, H., Litteral, J., Markey, M. K., Mercado, A., \& Scott, M. Adherence to Orthodontic Treatment in Youth With Cleft Lip and/or Palate. The Cleft Palate-Craniofacial Journal. 2019; 1055 66561986755. doi:10.1177/1055665619867556

17. Karp JM. Dental surgery under general anesthesia for preschool patients with orofacial clefts. Pediatr Dent 2009;31(5):426-431.

18. Crerand $C E$, Sarwer DB, Kazak AE, Clarke A, Rumsey $N$ Body image and quality of life in adolescents with craniofacial conditions. Cleft Palate Craniofac J. 2017;54(1):212.

19. Rosenberg J, Albert M, Aspinall C, Bautista S, Crilly Bellucci C, Edwards TC, et al. Parent Observations of the Health Status of Infants With Clefts of the Lip: Results From Qualitative Interviews. Cleft Palate Craniofac J. 2018; 9:1055665618793062.

20. Smedegaard LH, Marxen DR, Moes JH, Glassou EN, Scientsan C. Hospitalization, breast-milk feeding, and growth in infants with cleft palate and cleft lip and palate born in Denmark. Cleft Pal Craniofac J. 2008; 45(6):628632.

21. Zarate YA, Martin LJ, Hopkin R J, Bender PL, Zhang X, Saal $H M$. Evaluation of growth in patients with isolated cleft lip and/or cleft palate. Pediatrics. 2010; 125(3):e543e549.

22. Kaye A, Thaete K, Snell A, Chesser C, Goldak C, Huff H. Initial nutritional assessment of infants with cleft lip and/ or palate: interventions and return to birth weight. Cleft Palate Craniofac J. 2017; 54(2):127-136.

23. Baylis AL, Pearson GD, Hall C, Madhoun LL, Cummings C, Neal $N$, et al. A Quality Improvement Initiative to Improve Feeding and Growth of Infants With Cle$\mathrm{ft}$ Lip and/or Palate. Cleft Palate Craniofac J. 2018 Jan 1:1055665618. 